\title{
A systematic review of pharmacists' interventions to support medicines optimisation in patients with visual impairment
}

\author{
Basma Y. Kentab $^{1,2}$ (D) Heather E. Barry ${ }^{1}$ (D) $\cdot$ Sinaa A. Al-Aqeel ${ }^{2}$ (D) Carmel M. Hughes $^{1}$ (D)
}

Received: 28 May 2019 / Accepted: 7 September 2019 / Published online: 17 September 2019

(c) The Author(s) 2019

\begin{abstract}
Background People with visual impairment often report poorer health and encounter many challenges when using medicines. Pharmacists can play a significant role in optimising medicines use for these patients. However, little is known about pharmacists' current practices when providing services to this population nor the impact of such services, if any, on medicines optimisation-related outcomes. Aim of the review This systematic review aims to identify the types, and assess the effectiveness of, interventions provided by pharmacists on medicines optimisation-related outcomes. Method Systematic searches of the following electronic databases were carried out from date of inception to March 2018: Cochrane Library; MEDLINE; EMBASE; International Pharmaceutical Abstracts; Scopus; and Cumulative Index to Nursing and Allied Health Literature. Several trial registries and grey literature resources were also searched. Any randomised controlled trials, non-randomised controlled trials, controlled before-and-after studies, or interrupted time series analyses reporting on interventions provided by pharmacists to adult visually impaired patients and/or their caregivers in order to improve medicines optimisation-related outcomes of medicine safety, adherence, patient satisfaction, shared decision making, or quality of life were included. Results A total of 1877 titles/abstracts were screened, and 27 full text articles were assessed for eligibility. On examination of full texts, no studies met the inclusion criteria for this review. Conclusion This review highlights the need for future research that would be vital for promoting the safe and effective use of medicines and the delivery of pharmaceutical care services to people with visual impairment.
\end{abstract}

Keywords Medicines optimisation $\cdot$ Pharmacist $\cdot$ Systematic review $\cdot$ Visual impairment

\section{Impacts on practice}

- There is a lack of robust evidence to help identify the most effective interventions to optimise medicine use in people with visual impairment.

- The paucity of research on pharmacist-led medicines optimisation interventions may be explained by a num-

Electronic supplementary material The online version of this article (https://doi.org/10.1007/s11096-019-00907-2) contains supplementary material, which is available to authorized users.

Carmel M. Hughes

c.hughes@qub.ac.uk

1 Primary Care Research Group, School of Pharmacy, Queen's University Belfast, 97 Lisburn Road, Belfast BT9 7BL, Northern Ireland, UK

2 Department of Clinical Pharmacy, College of Pharmacy, King Saud University, Riyadh, Saudi Arabia ber of reasons relating to difficulties in implementing services in everyday practice as well as the various challenges to conducting research that involves people with visual impairment.

- With the expected increase in the numbers of visually impaired people due to population growth and ageing, there is a clear need for research into this area.

\section{Introduction}

Visual impairment has various definitions, none of which is universally agreed upon [1-4]. The World Health Organisation's (WHO) definition classifies visual impairment into seven categories from mild or no impairment to blindness based on presenting distance visual acuity [1]. Globally, an estimated 441 million people are considered visually impaired [5]. Of these, 36 million are categorized as blind, 
188 million have mild vision impairment, and 217 million have moderate to severe vision impairment.

Based on analysis of two large government surveys, the 2013 Sight Loss United Kingdom (UK) report has shown that more than half of people with sight loss were dissatisfied with their health [6]. In the United States (US), older people with vision problems were more likely to report their health as'poor' than people without sensory problems (15.3\% vs. $6.7 \%)$ [7]. Those with vision impairments were 3.1 times more likely to report difficulty taking medicines compared to those without sensory impairment [7]. Beverley et al. interviewed 31 visually impaired people and found that only a small proportion of health and social care needs were being met, and participants had many information needs including those related to techniques for administering medicines and reading medical information [8]. A recent scoping review of the pharmaceutical care needs of people with sensory loss (hearing and/or vision loss) highlighted the paucity of studies examining the pharmaceutical care needs of this population [9]. Challenges to medicines use experienced by those who are visually impaired include problems with distinguishing between medicines, doses and expiration dates as well as adherence issues [9-16]. Patients with visual impairment often need to rely on family members or carers to help with medicines [9].

Medicines optimisation is defined as "a person-centred approach to safe and effective medicines use, to ensure people obtain the best possible outcomes from their medicines" [17]. Evidence indicates that pharmacists can indeed have a significant impact on improving safe and effective medicines use for patients. An overview of systematic reviews found that some of the most promising interventions to improve medicines use outcomes were interventions involving pharmacists in medicines management and pharmaceutical care services [18]. In the field of disabilities, the literature has shown that pharmacists' interventions such as medicines review to identify drug therapy problems, can produce a positive impact on the quality of medicine use process for patients with intellectual disabilities [19].

Despite available guidance on methods for the provision of medicines information [20-23] and literature in the area of medicines use by visually impaired patients, little is known about the current level of visual impairment-specific pharmaceutical services provided, nor the impact of pharmacist-delivered services, if any, on medicines optimisationrelated outcomes.

\section{Aim of the review}

The aim of this review was to identify the types, and assess the effectiveness of, interventions provided by pharmacists on medicines optimisation-related outcomes in patients with visual impairment. The specific objectives were to determine:

- The types of interventions/services being provided by pharmacists to support medicines optimisation in patients with visual impairment; and

- The effectiveness of these interventions/services on medicines optimisation-related outcomes in patients with visual impairment.

\section{Ethical approval}

The study did not require approval of a review board.

\section{Method}

The review was guided by the recommendations of the Cochrane Handbook for Systematic Reviews of Interventions [24]. The reporting of the review complies with the Preferred Reporting Items for Systematic Reviews and Meta-Analyses statement [25]. The review protocol was registered with the International prospective register of systematic reviews (PROSPERO; registration number: CRD42018088776).

\section{Criteria for considering studies for this review}

Types of studies: All studies that met the Cochrane Effective Practice and Organization of Care Group [26] inclusion criteria were included in this review. These were randomised controlled trials (RCT), non-randomised controlled trials (NRCT), controlled before-and-after studies (CBA), and interrupted time series analyses (ITS). Other study designs were excluded because they cannot be used to establish causation between intervention and outcome. [26].

Types of participants: The participants included in this review were patients aged 18 years or over with visual impairment, as defined by the study investigators, and/or their caregivers who received medicine-related interventions or services delivered by pharmacists. The setting may have been an outpatient and/or inpatient setting in a hospital pharmacy, community pharmacy, primary healthcare centre, or long-term care facility. Patients younger than 18 years were excluded due to their unique needs and characteristics that are different from adults. There were no restrictions on the cause or degree of visual impairment, the medicines used (class of medicine, indication, number of medicines), or the number or type of concomitant diseases.

Types of interventions: Any interventions delivered by pharmacists that aimed to improve medicines optimisationrelated outcomes of medicine safety, adherence, patient 
satisfaction, shared decision making, or quality of life were identified. The interventions must have explicitly targeted visually impaired patients and/or their caregivers as primary recipients. Interventions targeting healthcare professionals or systems were excluded. There were no restrictions on the intervention duration or comparison group. Studies comparing intervention versus usual care, no intervention or alternative intervention were all eligible for inclusion. To help organize and provide a framework for selecting and categorizing these interventions, they were required to fall under one of the eight categories of the taxonomy developed by Lowe et al. [27].

Types of outcome measures: The outcomes of interest were based on the principles of medicines optimisation and involved changes in the following outcomes [17]: harm attributable to errors in medication (medication errors); patient satisfaction; quality of life; medication adherence; or shared decision making.

\section{Search strategy}

Six electronic bibliographic databases were searched from date of inception to March 2018 (Table 1). A comprehensive search strategy was developed in MEDLINE in consultation with an experienced librarian and the research team (Online Resource 1). The search strategy involved a combination of medical subject headings (MeSH) and keywords, relating to visual impairment, pharmacy and the outcomes of interest, as well as truncation adapted for the requirements of each database. The MEDLINE search strategy was adapted for other databases. The searches were conducted in English, but citations identified in any language were considered. In an effort to identify further published, unpublished, and ongoing trials, eight clinical trial registries and grey literature resources in addition to the websites of two non-profit organizations were searched for relevant studies (Table 1). The organizations were also contacted by email to help identify relevant research. Citation tracking using Web of Science Cited Reference Search for all included studies, if any, was also planned. Finally, references of included studies, if any, were to be hand-searched to identify additional studies.

\section{Data collection and analysis}

The references from all electronic searches were downloaded into EndNote X8.2 [28] and duplicates were removed. Titles and abstracts were screened for eligibility by one author (BK) with around $8 \%$ being independently screened by another author (SA). Studies were divided into three categories: 'definitely include', 'definitely exclude' and 'possibly include'. The full text copies of each study in the 'definitely include' or 'possibly include' categories were obtained. The full text copies were assessed independently by BK and SA for final eligibility using the inclusion/exclusion criteria, documenting reasons for exclusion. Disagreements regarding inclusion were resolved through discussion within the research team. We planned to have data extracted independently by two authors using a form derived from the data extraction template of the Cochrane Consumers and Communication Review Group [29], and to assess the risk of bias of included studies in accordance with the Cochrane Handbook for Systematic Reviews of Interventions [24] and the Cochrane Consumers and Communication guidelines [30] for each included study design. Studies were to be categorised as being at high, low or unclear risk of bias. Our decision to analyse data using meta-analysis was to be based
Table 1 Resources searched to identify potential studies from date of inception to March 2018

\begin{tabular}{ll}
\hline Electronic Databases & Cochrane Library \\
& MEDLINE (Ovid) \\
& EMBASE (Ovid) \\
International Pharmaceutical Abstracts (Ovid) & Scopus \\
& Cumulative Index to Nursing and Allied Health Literature (CINAHL) \\
ClinicalTrials.gov \\
$\begin{array}{l}\text { Grey Literature } \\
\text { Resources }\end{array}$ \\
\\
Australian New Zealand Clinical Trials Registry (ANZCTR) \\
WHO International Clinical Trials Registry Platform (ICTRP) \\
International Standard Randomized Controlled Trial Number (ISRCTN) Registry \\
International Prospective Register of Systematic Reviews (PROSPERO) \\
National Institutes of Health Clinical Centre Trials \\
UK Clinical Trials Gateway \\
Open Grey \\
The Royal National Institute of Blind People (RNIB) \\
American Foundation for the Blind (AFB)
\end{tabular}


on whether the interventions in included trials were similar enough in terms of participants, settings, interventions, comparison and outcome measures to ensure meaningful conclusions from a statistically pooled result. If data could not be pooled for meta-analysis, we planned to conduct a narrative synthesis of results. Data were to be grouped based on the category that would best explore the heterogeneity of studies and make most sense to the reader (such as by interventions, populations or outcomes).

\section{Results}

A total of 1877 studies were screened. Database searches identified 1189 studies after duplicates were removed. Of these, 1171 met the 'definitely exclude' category based on information in the title and abstract, leaving 18 studies identified as 'possibly include'. The search of other resources (i.e. trial registries and grey literature) identified 688 records. After initial screening, 679 records met the 'definitely exclude' category and nine were identified as 'possibly include'. No relevant studies were identified from RNIB or AFB.

On examination of the full text of the 27 studies in the 'possibly include' category, all were identified as not fulfilling the inclusion criteria for this review. The PRISMA flow chart shows the results for screening and selection of papers (Fig. 1) and Table 2 (Online Resource 2) lists the reason for exclusion for each study. There were four studies in which participants were not visually impaired. Fifteen studies did not fulfil the type of study criteria, the aim of the intervention in four studies was not medicines use, and the intervention was not delivered by pharmacists in one study. The full text could not be obtained for three records which meant that we could not confirm eligibility, hence they were excluded. There were no included studies for which to assess the risk of bias or to use for evidence synthesis.

\section{Discussion}

There were no studies that met the inclusion criteria for this review, which was not unexpected. The review by Killick et al., that was published shortly after our search had been completed, highlighted the lack of intervention studies to enhance pharmaceutical care for people with sensory loss [9]. The systematic review by Beverly et al. [31] on health information needs of visually impaired people identified only a few studies, most of which were of poor quality. When evaluating the literature, over a 20 years period, on interventions implemented by pharmacists to improve the care of people with intellectual disabilities, O'Dwyer et al. [19] identified only two intervention studies, and three reports of pharmacists' involvement in service provision as part of a multi-disciplinary team. Neither of the intervention studies were randomised controlled trials.

There are several explanations for the limited research in this area. The burden of visual impairment is considered greatest in those aged 50 years and older (with this group accounting for $86 \%$ of blind people and $80 \%$ of people with moderate-severe visual impairment) [32]. Visual impairment is also considered the leading cause of age-related disability [33]. For these reasons, challenges encountered in involving both older people and those with disabilities in research may further complicate the picture. Indeed, several reports indicate that older adults are often under-represented in health research and various methods for enhancing their involvement, recruitment and retention are suggested [34-40]. Moreover, despite having a higher incidence of chronic conditions and health-related disparities, people with disabilities are mostly absent from mainstream health research because of overly rigid exclusion criteria and lack of consideration for proper accessibility and accommodation in research design [41].

Beadle-Brown et al. [42] reviewed the barriers and facilitators to the involvement of 'seldom-heard' groups in research. One of the barriers identified was assumptions made by researchers that these groups' views were not possible to access perhaps because of communication difficulties, or that their views were not valid or worthy of consideration [42]. Duckett and Pratt referred to what one participant described as visually impaired people 'staying in the woodwork', i.e. not highlighting their needs [43]. People also indicated that they may not wish to participate in research if that meant they had to be identified as 'visually impaired' or associate with other visually impaired people, and they had concerns about the need to understand the diversity of visually impaired people in terms of their degree of impairment, strengths, needs and aspirations [43].

Finding and recruiting 'seldom-heard' participants including older people and those who are visually impaired can be problematic due to: lack of interest of potential participants in the research area; difficulties understanding study information and requirements; inaccessible research venues; and a lack of relevant support such as transport [34, 35, 38, 42].

External oversight of studies may also be a challenge. The need to obtain ethical approval which may be associated with inflexible procedures related to gaining consent or standard information sheets may prevent the inclusion of particular groups [42]. The role of gatekeepers, such as medical practitioners or caregivers in protecting or selecting potential participants may create further barriers to recruitment [42]. Moreover, people may not trust the person or 


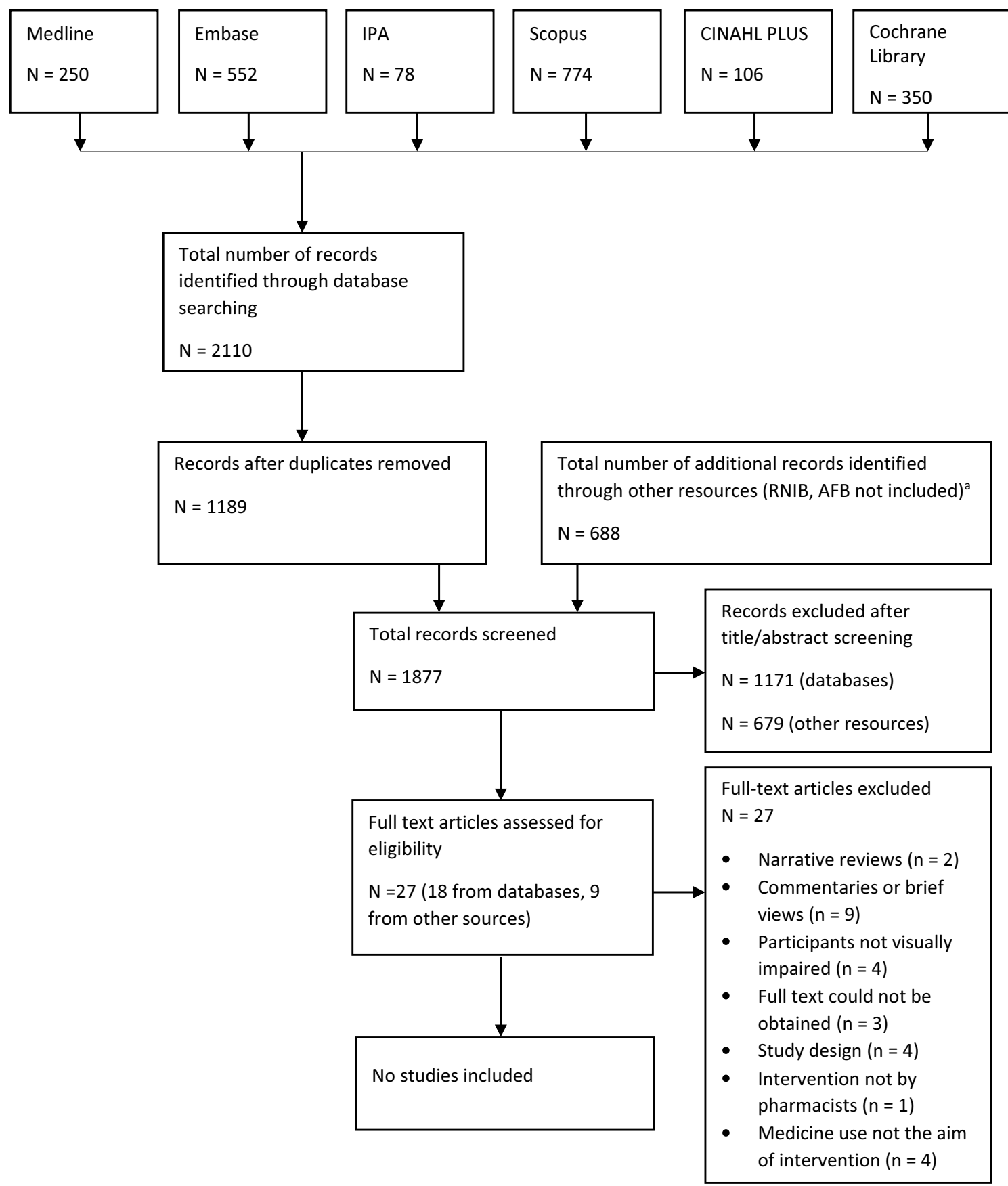

a It was not possible to combine and remove duplicates from searching trial registries and grey literature resources because some of them did not have the option to import results.

Fig. 1 Preferred reporting items for systematic reviews and meta-analyses (PRISMA) flow diagram of screening process and reasons for exclusion of studies

institution asking them to take part in a study because of past negative experiences; they may have concerns about appearing uneducated in comparison to the researcher or have concerns about the proposed level of privacy and confidentiality $[34,35,38,42]$. Another issue is the power dynamics of research settings where participants are expected to have a compliant passive role, but wish for a more empowering relationship that would allow them to question researchers and satisfy their own information needs [43]. Initiating contact through a trusted person and her willingness to answer a number of questions for the participants about her credentials helped Moore gain permission from several visually 
impaired participants to participate in an interview-based study [44].

Barriers may also arise from instruments used for data collection. Lengthy surveys, complex or irrelevant questions in questionnaires or interviews, or utilizing instruments without validity testing may be problematic $[42,45]$. The lack of data on the prevalence of visual impairment and details about the affected persons, and absence of a coordinating research body or consortium to organize and prioritise the research efforts and funding may also constitute significant challenges to research [46].

Being aware of the research needs of people with visual impairment should play a major role in guiding future research. Patients with visual impairment have traditionally been involved in research as 'subjects' [31], but evidence indicates that they want greater inclusion in research and for such research to be more empowering and emancipatory [43]. The attempt by Schilling and Gerhardus to review methods for involving older people with visual impairment in health research carried out 'with' or 'by' them rather than 'about' or 'for' them failed to identify any relevant studies [40]. International experts from five research groups in geriatric pharmacotherapy, including five pharmacists, considered the use of medicines by frail and vulnerable patient groups, one of the research priorities for optimising geriatric pharmacotherapy [47]. When consulted about their opinions on topics to be researched, people with visual impairment identified the access to information including these related to diagnosis and treatment as one of the topics that warranted further attention [43]. In the Netherlands, improvement of visual impairment treatments and technologies (e.g. approaches to improve conversion of written text to accessible format) were prioritized highly on the list of research topics compiled after seeking opinions of people with visual impairment [48]. Moreover, improving adherence to glaucoma treatment was considered one of the ten glaucoma research priorities by patients, carers and clinicians in the UK Sight Loss and Vision Priority Setting Partnership [49].

Many difficulties may prevent the development and implementation of interventions to optimise medicines use for patients with visual impairment. These include the lack of time, staff, sufficient training and availability of assistive technology to produce accessible information, as well as the lack of the awareness of pharmacy staff of the needs of people with visual impairment $[16,50,51]$. One reason for pharmacists' lack of awareness may be their failure to identify such patients in everyday practice. This may be caused by ineffective communication and visual impairment being not necessarily visible. Visually impaired patients may also not wish to disclose their disability or may rely on family members or carers [11,13].

This review has highlighted the need for further research that focuses initially on exploring the opinions and views of key stakeholders (e.g. visually impaired patients and pharmacists) utilizing a variety of qualitative and quantitative methods. These will inform subsequent work on designing, implementing and evaluating interventions to optimise medicines use. Several recommendations may be useful to minimize the barriers to research involving people with visual impairment such as engaging with proxy respondents (e.g. carers) and being adequately resourced with time and money to make use of alternative formats for providing information and to recruit participants [41-45, 52]. Telephone interviews may be useful to increase flexibility and provide a more comfortable setting for participants [45].

\section{Strengths and limitations}

This study is the first to review and synthesise medicines optimisation interventions provided by pharmacists for people with visual impairment. We used a number of definitions of visual impairment in our search which facilitated identification of a number of papers. Comprehensive searches of multiple databases, trial registries and other sources were undertaken, but it is still possible that some unpublished or unindexed data might have been missed. The review specified certain study designs (RCT, NRCT, CBA, and ITS) aiming to identify only high quality evidence which resulted in no studies meeting the inclusion criteria. The review did not consider evidence pertaining to those under the age of 18 .

\section{Conclusion}

This systematic review identified no eligible studies on interventions provided by pharmacists to support medicines optimisation in patients with visual impairment based on our inclusion criteria. There is a clear need for future studies to explore the type of interventions that could support medicines optimisation in these patients. Testing and comparing the effectiveness of different interventions using robust study designs is also essential.

Acknowledgements The authors would like to acknowledge the valuable input of Ms. Angela Thompson (Queen's University Belfast Library) in development of the search strategy.

Funding Basma Kentab is supported by a PhD Scholarship from the Saudi Arabian Government. The funder played no role in the design, analysis or conduct of the review.

Conflicts of interest The authors declare that they have no conflict of interest.

Open Access This article is distributed under the terms of the Creative Commons Attribution 4.0 International License (http://creativeco mmons.org/licenses/by/4.0/), which permits unrestricted use, distribution, and reproduction in any medium, provided you give appropriate 
credit to the original author(s) and the source, provide a link to the Creative Commons license, and indicate if changes were made.

\section{References}

1. World Health Organization. International statistical classification of diseases and related health problems. 2016. http://apps.who. int/classifications/icd10/browse/2016/en\#/H54.9. Accessed 10 Jan 2018.

2. Dementia and Disabilities Unit, Social Care, Ageing and Disability Directorate. Certificate of vision impairment: Explanatory notes for consultant ophthalmologists and hospital eye clinic staff in England. Department of Health. 2017. https://www.gov.uk/ government/uploads/system/uploads/attachment_data/file/63759 0/CVI_guidance.pdf. Accessed 6 Feb 2018.

3. National Eye Institute. Definitions. n.d. https://nei.nih.gov/eyeda ta/defined. Accessed 10 Jan 2018.

4. National Eye Institute. Low vision glossary. n.d. https://nei.nih. gov/lowvision/content/glossary. Accessed 10 Jan 2018.

5. World Health Organization. Blindness and vision impairment fact sheet. 2018. https://www.who.int/news-room/fact-sheets/detail/ blindness-and-visual-impairment. Accessed 15 Jan 2019.

6. Evidence and Service Impact Team. Sight loss UK 2013: the latest evidence. Royal National Institute of Blind People. 2013. http:// www.rnib.org.uk/knowledge-and-research-hub/research-reports/ general-research. Accessed 10 Jan 2018.

7. Crews JE, Campbell VA. Vision impairment and hearing loss among community dwelling older Americans: implications for health and functioning. Am J Public Health. 2004;94(5):823-9.

8. Beverley CA, Bath PA, Barber R. Health and social care information for visually-impaired people. Aslib Proc. 2011;63(2/3):256-74.

9. Killick K, Macaden L, Smith A, Kroll T, Stoddart K, Watson MC. A scoping review of the pharmaceutical care needs of people with sensory loss. Int J Pharm Pract. 2018;26(5):380-6.

10. Riewpaiboon A. How the blind cope with problems of medicine utilization: a study in Bangkok, Thailand. Pharmacoepidemiol Drug Saf. 2009;18(8):708-12.

11. McCann RM, Jackson AJ, Stevenson M, Dempster M, McElnay JC, Cupples ME. Help needed in medication self-management for people with visual impairment: case-control study. Br J Gen Pract. 2012;62(601):e530-7.

12. Weeraratne $\mathrm{C}$, Opatha $\mathrm{S}$, Rosa C. Challenges faced by visually disabled people medicines in use of medicines, self-adopted coping strategies and medicine-related mishaps. WHO South East Asia J Public Health. 2012;1(3):256-67.

13. Kentab BY, Al-Rowiali KZ, Al-Harbi RA, Al-Shammari NH, Balhareth WM, Al-Yazeed HF. Exploring medication use by blind patients in Saudi Arabia. Saudi Pharm J. 2015;23(1):102-6.

14. Apoorva R, Vasundara K, Umadevi R. A study on medication adherence, medication related challenges faced and coping strategies adopted by visually impaired subjects in a tertiary care hospital. Int J Basic Clin Pharmacol. 2017;6(10):2323-7.

15. Zhi-Han L, Hui-Yin Y, Makmor-Bakry M. Medication-handling challenges among visually impaired population. Arch Pharm Pract. 2017;8(1):8-14.

16. Alhusein N, Macaden L, Smith A, Stoddart KM, Taylor AJ, Killick K, et al. 'Has she seen me?': a multiple methods study of the pharmaceutical care needs of older people with sensory impairment in Scotland. BMJ Open. 2018;8(8):e023198.

17. National Institute for Health and Care Excellence. Medicines optimisation quality standard. 2016. https://www.nice.org.uk/guida nce/qs120. Accessed 10 Jan 2018.
18. Ryan R, Santesso N, Lowe D, Hill S, Grimshaw J, Prictor M et al. Interventions to improve safe and effective medicines use by consumers: an overview of systematic reviews. Cochrane Database Syst Rev. 2014(4):CD007768.

19. O'Dwyer M, Mestrovic A, Henman M. Pharmacists' medicinesrelated interventions for people with intellectual disabilities: a narrative review. Int J Clin Pharm. 2015;37(4):566-78.

20. American Society of Consultant Pharmacists Foundation and American Foundation for the Blind. Guidelines for prescription labelling and consumer medication information for people with vision loss. American Foundation for the Blind. 2008. http:// elearn.afb.org/section.aspx?FolderID $=3 \&$ SectionID $=3 \&$ Topic $\mathrm{ID}=403 \&$ SubTopicID $=256 \&$ DocumentID $=4064$. Accessed 11 Jan 2018.

21. European Commission. Guidelines on the readability of the labelling and package leaflet of medicinal products for human use. 2009. https://ec.europa.eu/health//sites/health/files/files/eudralex/ vol2/c/2009_01_12_readability_guideline_final_en.pdf. Accessed 11 Jan 2018.

22. Access Board Working Group on Accessible Prescription Drug Container Labels. Best practices for making prescription drug container label information accessible to persons who are blind or visually-impaired or who are elderly. United States Access Board. 2013. https://www.access-board.gov/guidelines-and-stand ards/health-care/about-prescription-drug-container-labels/worki ng-group-recommendations. Accessed 7 Feb 2018.

23. NHS England. Accessible information standard. resources: factsheets and clarifying notes. 2016. https://www.england.nhs. uk/ourwork/accessibleinfo/resources/. Accessed 6 Feb 2018.

24. Higgins J, Green S. Cochrane handbook for systematic reviews of interventions. Version 5.1.0 ed. The Cochrane Collaboration; 2011.

25. Moher D, Liberati A, Tetzlaff J, Altman DG, Group P. Preferred reporting items for systematic reviews and meta-analyses: the PRISMA statement. PLoS Med. 2009;6(7):e1000097.

26. Cochrane Effective Practice and Organisation of Care (EPOC). What study designs can be considered for inclusion in an EPOC review and what should they be called. 2017. http://epoc.cochr ane.org/resources/epoc-resources-review-authors. Accessed 7 Feb 2018.

27. Lowe D, Ryan R, Santesso N, Hill S. Development of a taxonomy of interventions to organise the evidence on consumers' medicines use. Patient Educ Couns. 2011;85(2):e101-7.

28. Clarivate Analytics. Endnote. Version X8.2. Philadelphia, PA: Clarivate Analytics; 2018.

29. Ryan R, Synnot A, Prictor M, Hill S. Data extraction template for included studies. Cochrane Consumers and Communication Group. 2016. http://cccrg.cochrane.org/author-resources. Accessed date $7 \mathrm{Feb} 2018$.

30. Ryan R, Hill S, Prictor M, McKenzie J. Study quality guide. Cochrane Consumers and Communication Group. 2013. http:// cccrg.cochrane.org/author-resources. Accessed date 7 Feb 2018.

31. Beverley C, Bath P, Booth A. Health information needs of visually impaired people: a systematic review of the literature. Health Soc Care Community. 2004;12(1):1-24.

32. Bourne RRA, Flaxman SR, Braithwaite T, Cicinelli MV, Das A, Jonas JB, et al. Magnitude, temporal trends, and projections of the global prevalence of blindness and distance and near vision impairment: a systematic review and meta-analysis. Lancet Glob Health. 2017;5(9):e888-97.

33. International Federation on Ageing. The high cost of low vision: The evidence on ageing and the loss of sight. 2013. http://www. ifa-fiv.org/wpcontent/uploads/2013/02/The-High-Cost-of-LowVision-The-Evidence-on-Ageing-and-the-Loss-of-Sight.pdf. Accessed 8 Feb 2019. 
34. Mody L, Miller DK, McGloin JM, Freeman M, Marcantonio ER, Magaziner J, et al. Recruitment and retention of older adults in aging research. J Am Geriatr Soc. 2008;56(12):2340-8.

35. Ridda I, MacIntyre CR, Lindley RI, Tan TC. Difficulties in recruiting older people in clinical trials: an examination of barriers and solutions. Vaccine. 2010;28(4):901-6.

36. McMurdo ME, Roberts H, Parker S, Wyatt N, May H, Goodman $\mathrm{C}$, et al. Improving recruitment of older people to research through good practice. Age Ageing. 2011;40(6):659-65.

37. McHenry JC, Insel KC, Einstein GO, Vidrine AN, Koerner KM, Morrow DG. Recruitment of older adults: success maybe in the details. Gerontologist. 2015;55(5):845-53.

38. Knechel N. The challenges of enrolling older adults into intervention studies. Yale J Biol Med. 2013;86(1):41-7.

39. Lacey RJ, Wilkie R, Wynne-Jones G, Jordan JL, Wersocki E, McBeth J. Evidence for strategies that improve recruitment and retention of adults aged 65 years and over in randomised trials and observational studies: a systematic review. Age Ageing. 2017;46(6):895-903.

40. Schilling I, Gerhardus A. Methods for involving older people in health research-a review of the literature. Int J Environ Res Public Health. 2017;14(12).

41. Rios D, Magasi S, Novak C, Harniss M. Conducting accessible research: including people with disabilities in public health, epidemiological, and outcomes studies. Am J Public Health. 2016;106(12):2137-44.

42. Beadle-Brown J, Ryan S, Windle K et al. Engagement of people with longterm conditions in health and social care research: Barriers and facilitators to capturing the views of seldom-heard populations. Quality and Outcomes of Person-Centred Care Policy Research Unit. 2012. https://www.qoru.ac.uk/wp-content/uploa ds/2013/02/E1_1-Report-ID4373.pdf. Accessed 12 Jan 2019.

43. Duckett PS, Pratt R. The researched opinions on research: visually impaired people and visual impairment research. Disabil Soc. 2001;16(6):815-35.

44. Moore L. Conducting research with visually impaired older adults. Qual Health Res. 2002;12(4):559-65.
45. Trujillo Tanner C, Caserta MS, Kleinschmidt JJ, Clayton MS, Bernstein PS, Guo JW. Conducting research with older adults with vision impairment: lessons learned and recommended best practices. Gerontol Geriatr Med. 2018;4:2333721418812624.

46. Welp A, Woodbury RB, McCoy MA, Teutsch SM, editors. Making eye health a population health imperative: vision for tomorrow. Washington, DC: National Academies Press; 2016.

47. Tan ECK, Sluggett JK, Johnell K, Onder G, Elseviers M, Morin $\mathrm{L}$, et al. Research priorities for optimizing geriatric pharmacotherapy: an international consensus. J Am Med Dir Assoc. 2018;19(3):193-9.

48. Schölvinck A, Pittens C, Broerse J. The research priorities of people with visual impairments in the Netherlands. J Vis Impair Blind. 2017;111(3):201-17.

49. Rowe F, Wormald R, Cable R, Acton M, Bonstein K, Bowen M, et al. The sight loss and vision priority setting partnership (SLVPSP): overview and results of the research prioritisation survey process. BMJ Open. 2014;4(7):e004905.

50. Sibley E. Towards an inclusive health service: A report into the availability of health information for blind and partially sighted people. Royal National Institute of Blind People. 2009. http:// www.rnib.org.uk/knowledge-and-research-hub/research-reports/ general-research/towards-inclusive-health. Accessed 11 Jan 2018.

51. Barnett N, Bushra A, Huddy H, Majekodumni F, Thomas S, Chiu F. How to support patients with sight loss in pharmacy. Pharm J. 2017;299(7904):1-8.

52. Skilton A, Boswell E, Prince K, Francome-Wood P, Moosajee M. Overcoming barriers to the involvement of deafblind people in conversations about research: recommendations from individuals with usher syndrome. Res Involv Engagem. 2018;4:40.

Publisher's Note Springer Nature remains neutral with regard to jurisdictional claims in published maps and institutional affiliations. 